\title{
El Desarrollo del Hardware para la Realidad Virtual
}

\author{
Graciela Lara ${ }^{1}$, Alexis Santana ${ }^{1}$, Andrés Lira ${ }^{1}$, Adriana Peña $^{1}$ \\ graciela.lara@academicos.udg.mx, alexis.s.g@hotmail.com, \\ Adriana.peña@cucei.udg.mx \\ eliteandryod@gmail.com, \\ ${ }^{1}$ 1CUCEI, Universidad de Guadalajara, Blvd. Marcelino García Barragán 1421, C.P. 44430, Guadalajara, \\ Jalisco, México.
}

DOI: 10.17013/risti.31.106-117

\begin{abstract}
Resumen: La Realidad Virtual (RV) es una simulación de un Ambiente Virtual (AV) creado por computadora en el que el usuario tiene la sensación de estar dentro de ese entorno, al mismo tiempo que puede interactuar usando tecnología de hardware llena de posibilidades, con aplicaciones en múltiples áreas tales como la medicina, entretenimiento, educación, psicología, comercio electrónico, entre muchas otras. Con el apoyo de dispositivos como cascos de Realidad Virtual, gafas, y posicionadores, entre otros es posible alcanzar una inmersión total y una mayor interacción dentro de estos ambientes. Asimismo, el usuario puede experimentar que desaparece del mundo real. En este trabajo profundizamos sobre el desarrollo y la evolución de las herramientas de hardware que han permitido la interacción entre los usuarios y los entornos virtuales en $3 \mathrm{D}$. Al mismo tiempo, proponemos una clasificación en eras para evolución de los dispositivos de realidad virtual.
\end{abstract}

Palabras-clave: Entorno Virtual; Dispositivos de RV; IHC; Inmersión.

\section{The Hardware Development for Virtual Reality}

\begin{abstract}
Virtual Reality (VR) is a simulation of a Virtual Environment (VE) created by computer in which the user has the sensation of being inside that environment, at the same time that can interact using hardware technology full of possibilities, with applications in multiple areas such as medicine, entertainment, education, psychology, e-commerce, among many others. With the support of devices such as Virtual Reality helmets, glasses, and positioners, among others it is possible to achieve total immersion and greater interaction within these environments. Also, the user may experience that it disappears from the real world. In this work, we go deeper into the development and evolution of hardware tools that have allowed the interaction between users and virtual environments in $3 \mathrm{D}$. At the same time, we propose a classification in eras for the evolution of virtual reality devices.
\end{abstract}

Keywords: Virtual Environment; VR devices; HC; Immersion

\section{Introducción}

Dentro de la Realidad Virtual, no sólo basta disponer de las herramientas de software, sino que además es indispensable el uso de dispositivos (hardware), que logren introducir 
al usuario en un entorno virtual. Hardware es una palabra de origen inglesa que la Real Academia Española define como el conjunto de aparatos o dispositivos auxiliares e independientes de una computadora.

Los dispositivos periféricos (hardware) en la Realidad Virtual, permiten al usuario estimular sus procesos sensoriales, principalmente el de la vista, el oído y el tacto (Islas, Zabre \& Pérez, 2004). En este sentido, dentro de las aplicaciones de Realidad Virtual es importante incluir el manejo del hardware de interacción que sea adecuado, simple de manejar e intuitivo para conseguir el vínculo entre lo real y lo que se quiere percibir como real.

Disponer de hardware conveniente para el uso de aplicaciones de RV, ha permitido alcanzar una retroalimentación e interpretación del comportamiento de usuario. Un ejemplo de estas aplicaciones es el que presentan (Arango, Mazo, Palacio, 2013), donde es posible detectar señales neuronales, para conocer la intención de cerrar o abrir un modelo virtual de una mano o prótesis pegada al muñón real de un usuario. Para reconocer este proceso, se utilizó un dispositivo BCI (por sus siglas en inglés Brain Computer Interfaces), conocido como Emotiv EPOC. La disposición de hardware brinda rapidez y rendimiento necesario para obtener la mejor experiencia de RV.

Evidentemente, la disposición de hardware viene vinculada desde la capacidad grafica de los ordenadores y sus componentes. Estos componentes contemplan un procesador con una frecuencia y número de núcleos capaces, para atender la cantidad de procesos que puedan ejecutarse de manera sincrónica; la memoria RAM necesaria para ejecutar otras aplicaciones en segundo plano; las unidades de almacenamiento, donde se montarán las aplicaciones de RV; así como otro software necesario para su ejecución. Finalmente, es indispensable disponer de una tarjeta gráfica poderosa, para que el rendimiento de las aplicaciones de RV sea eficaz.

Dentro de este contexto, los tipos de dispositivos periféricos se han clasificado en dispositivos de entrada y dispositivos de salida $(E / S)$. Los primeros se ocupan de captar los movimientos de los usuarios en los EVs y de dirigirlos al computador; como parte de estos dispositivos se encuentran los posicionadores (sistemas de tracking), guantes, micrófonos, ratones, andadores, mesas de visualización, entre muchos otros. Los segundos tiene la función de convertir las señales de audio, video y demás derivados del computador en un estímulo, para los sentidos de los usuarios en Ambientes Virtuales (AVs); aquí se pueden identificar dispositivos como los displays basados en proyector (CAVE), plataformas móviles, sistemas de sonido, altavoces, entre otros. Ambos tipos de dispositivos logran la comunicación entre el usuario y los AVs.

Desde una perspectiva más práctica y como parte de los efectos que estos dispositivos pueden provocar en los usuarios, también se pueden catalogar lo dispositivos para la RV en de visión, de audición, y para interactuar. Los primeros facilitan una visión similar a la real, a través de displays con una vista estereoscópica. Los segundos emiten sonidos propios de los EVs que dependiendo de punto de ubicación estimulan el sentido del oído de los usuarios. Finalmente, los últimos consideran dispositivos tanto de entrada como de salida, debido a que proporcionan al usuario la interacción con el AV, a través de movimientos que pueden ser rastreados.

Como antecedentes se reconoce que el teclado y el ratón fueron los dispositivos básicos, para la comunicación e interacción entre humanos y computadoras desde la invención 
del primer ordenador personal manejado a ratón por Charles Thacker (Wadlow, 1981) considerandos, por lo tanto, los dispositivos que permitieron a los usuarios sumergirse en AVs. Sin embargo, antes el uso del ratón como un dispositivo elemental de interacción, se diseñó y desarrollo hardware para la interacción con ambientes específicos, creados principalmente para proyecto militares, siendo estos dispositivos extensamente caros y no multipropósito.

Sin duda, una característica en común de estos dispositivos de interacción con la computadora y los AVs ha sido la inmersión. Estaban diseñados principalmente para crear la interacción humano-computador tan natural como fuese posible. Esto a su vez, mejoro altamente una de las principales características de los AVs, la cantidad de presencia que evocan en sus usuarios (Meehan, Insko, Whitton \& Brooks, 2002).

A través de los años, estos y otros dispositivos han sido incorporados y aceptados de forma masiva entre los usuarios de AVs, para proporcionales la ilusión de presencia física. Por otro lado, las tecnologías de fabricación has evolucionado, permitiendo ser capaces de competir entre fabricantes en términos de calidad, rendimiento y costos.

La mayoría de estos dispositivos, también han avanzado con mejoras en el aspecto de ergonomía. Aspecto importante en el diseño del hardware, el cual se centra en la protección de la salud de los usuarios, desde distintos enfoques tales como: el agrado, la comodidad, la eficiencia, la eficacia, la seguridad, y el bienestar de los usuarios (Ver figura 1). Asimismo, esta evolución ha generado un cambio en la adaptación dentro de un mundo constantemente variable, donde las demandas de los usuarios no se dejaban esperar.

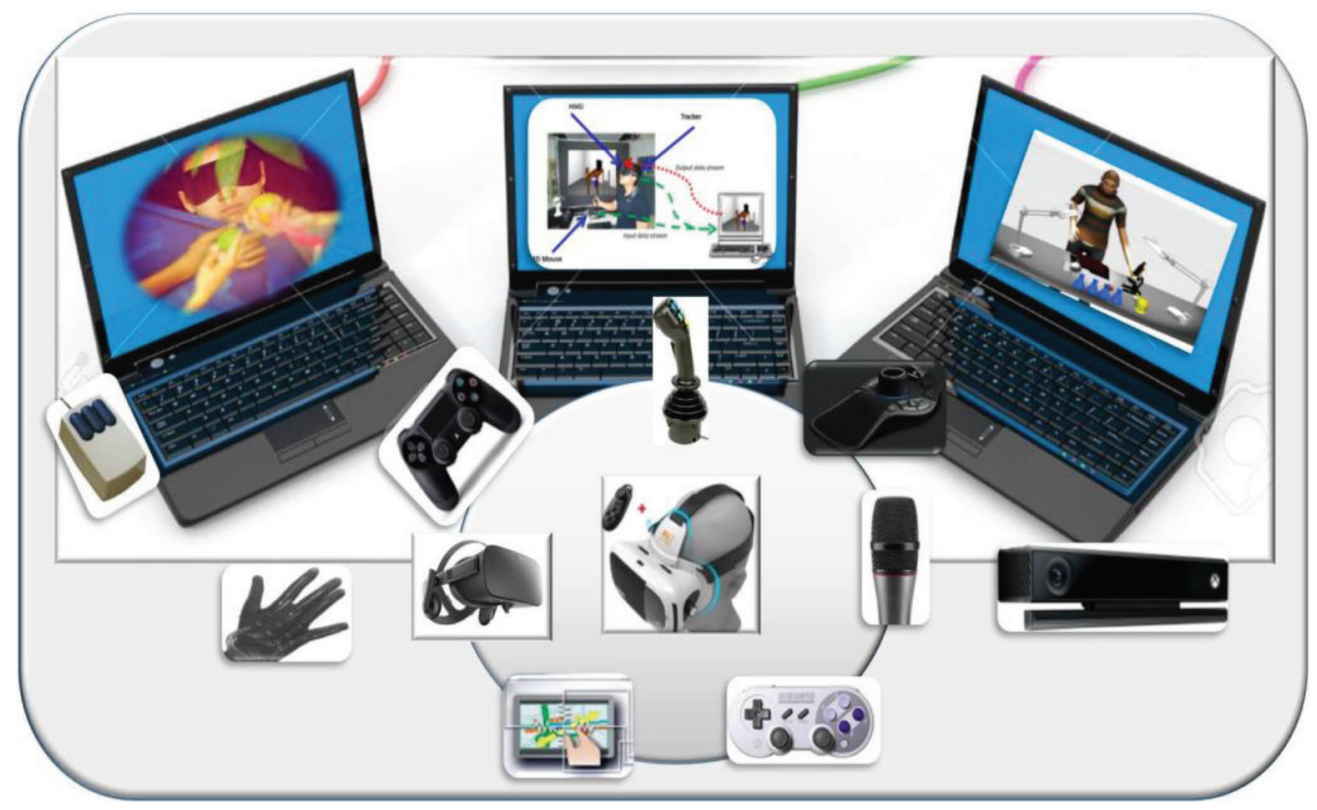

Figura 1 - Dispositivos usados en la Realidad Virtual 
Cabe menciona, algunos dispositivos desarrollados nunca fueron bien recibidos por la audiencia o su tecnología no era apreciada hasta hace algunos años. La rueda de desplazamiento, inventada para la armada británica para manipular una computadora utilizada para atacar aeronaves en 1946 (Engineering and Technology History Wiki, 2015), es un ejemplo de dichas innovaciones, trascendiendo como concepto al hardware de hoy en día como el SpaceTraveler de 3Dconnexion (3DConnexion, 2003) y SpaceNavigator (3DConnexion, 2007), entre otros más productos fabricados por la empresa 3Dconnexion, quien en la actualidad sigue desarrollando tecnología de navegación para AVs 3D y aplicaciones CAD (Diseño Asistido por Computadora) como SpanceNavigator, Cadmouse, SpaceMouse Pro. Dispositivos reconocidos como controladores de movimiento compatibles con varias aplicaciones tales como: Blender, Photoshop, Second Life, Virtual Earth 3D, entro otras (3dconnexion, 2018). Podemos observar otro gran ejemplo en la invención del joystick, creado en 1926 para pilotar aeronaves no tripuladas (NRL review 2011, 2011), pero no fue completamente utilizado hasta 1944 para dirigir cohetes y misiles (Wolf, 1997), dicha tecnología se sigue utilizando hoy en día en una variedad de joysticks utilizados principalmente en juegos.

A través delos años han sido varias las características consideradas en el diseño, desarrollo y uso de los dispositivos de E/S, para interactuar con los AVs, las cuales nos permiten clasificarlos en tres eras: la era Precursora, era Prototipo y la era Del Consumidor.

\section{Era Precursora}

La era Precursora toma lugar de 1940 a 1968 y se caracterizaba por el inmenso equipo de cómputo utilizado para interactuar con la realidad virtual. Sus costos de producción altamente caros y la prioridad militar y privada de los proyectos son otras características consideradas.

Durante este tiempo la mayoría de dispositivos E/S eran creados con una meta militar. Tal era el caso del joystick, creado con la intención de dirigir misiles a sus objetivos (Wolf, 1997) y manipular aeronaves no tripuladas. Este dispositivo se caracterizaba por ser un controlador en el que un par de controladores expedían ráfagas de señales con un mismo canal de comunicación, a través de un rango de frecuencia de rayos infrarrojos, con una transmisión electromagnética sin pérdida de datos reconocibles (Leung, 1990). Esta tecnología al final de este mismo periodo, fue adaptada para funcionar como un control para una consola de televisión llamada Magnavox Odyssey (Huhtamo, 2007) y después fue producida para una audiencia más amplia.

Otro ejemplo de los dispositivos E/S desarrollados en este periodo es el proyecto Super Cockpit creado por Thomas Furness III (Furness, 1986). El cual era esencialmente una réplica de la cabina de una aeronave pilotada remotamente mediante gestos con las manos. Furness a finales de la década de los 70 ya comenzaba el desarrollo de interfaces virtuales, para tener el mando de vuelos. En este proyecto incorpora la tecnología HDM como simulador de entrenamiento, y agrega además el seguimiento de la cabeza, los ojos, así como el reconocimiento de voz (Enciclopedia Británica, 2019).

Durante esa época un considerable número de proyectos surgieron, aunque la mayoría de ellos eran altamente secretos y no se considera que hayan tenido una especial relevancia. 
Sin embargo, todos estos crearon dispositivos de interacción únicos y personalizados. Estos dispositivos E/S intentaron asegurar un alto nivel de inmersión para los soldados involucrados, ayudándolos a interactuar en un ambiente semi-real con el objetivo de ayudarlos en su preparación y entrenamiento para situaciones en la vida real.

Por otra parte, la industria del entretenimiento observo las invenciones de Morton Heilig quien, en 1962, invento el Sensorama la cual era una maquina en forma de caja con una silla motorizada y una cabina que cubría el rostro del usuario. Era un dispositivo mecánico grande, voluminoso (Arce, 2013), que contaba con una pantalla estereoscópica a color, ventiladores, emisores de olor, sistema de sonido estéreo y poseía instalados cinco cortos cinematográficos sincronizados con el hardware para estimular los sentidos del usuario mediante sus diferentes métodos (Heilig, 1962). Este dispositivos tuvo un alto costo y dificultad para su fabricación, por lo cual no fue puesta para producida en serie (Martín-Herrera, 2012). Heilig además invento la Telesphere Mask, una máscara redonda que soportaba imágenes con soporte de visión periférica del 100\% y sonido biaural (Heilig, 1998).

No muy lejos, en 1968, Ivan Sutherland y Bob Sproull crearon The Sword of Damocles una inmensa máquina con una pantalla que colgaba del techo debido a que poseía un gran peso, este se colocaba en la cara del usuario. Una computadora utilizaba la posición de la cabeza para computar los elementos del escenario en base a la matriz de rotación y traslación y generar una vista apropiada en relación al punto de vista particular (Sutherland, 1968). Esta máquina también era capaz de rastrear tanto la posición del usuario como la posición de sus ojos a fin de actualizar la vista estereoscópica de acuerdo a la posición del usuario (Boas, 2013).

La mayoría de estos dispositivos requerían una gran parte de un cuarto y un inmenso equipo de cómputo para poder ser utilizados. Sus costos de producción eran alrededor de los cientos de millones de dólares, haciendo prácticamente imposible el ser usado con propósitos personales, con la excepción del joystick (Huhtamo, 2007).

\section{Era Prototipo}

Prototipo abarca de 1970 hasta 1995 y es caracterizada por equipo de cómputo más accesible, cambiando todos los equipos y máquinas especializadas por computadoras de escritorio y reduciendo los costos de producción permitiendo a las universidades y grandes industrias experimentar e intentar crear sus propios dispositivos E/S, para una interacción con un mayor factor de inmersión.

Durante este período, los beneficios de los ambientes virtuales comenzaron a llamar la atención de diferentes campos como tratamientos médicos, apoyo psicológico, tratamientos de recuperación, diseño de automóviles y videojuegos caseros. Por extensión, esto significo la evolución para los dispositivos de interacción en los ambientes virtuales.

Para algunos diseños y creaciones emergentes se tomó una aproximación medica como el Sayre Glove desarrollado por Thomas DeFanti y Daniel Sandin en la Universidad de Illinois en Chicago, este es un económico y ligero guante para monitorear movimiento de la mano, basado en la idea de Rich Sayre a quien se le acredita el nombre. Estos 
se utilizaron como un método efectivo de control multidimensional, además de una interacción más humana dentro de ambientes virtuales, utilizando literalmente sus manos como método primario de interacción (Sturman \& Zeltzer, 1994).

Otro dispositivo que hizo su debut en este periodo fue el primer gamepad moderno por la Nintendo TM Company Ltd. para su consola casera en 1983. Poseía un grupo de cuatro botones en forma de cruz utilizados para propósitos direccionales en el AV además de dos botones utilizados como botones de acción. El cual se volvió un estándar para gamepads como dispositivos de interacción en épocas recientes. Combinando los joysticks con sets de botones como el gamepad original de Nintendo creó gamepads que permitieron un movimiento más natural dentro de un AV. Esto facilito una inmersión más profunda dentro de los AVs.

En 1993 la compañía LogiCad3D TM (ahora 3Dconnexion TM) creo si primer ratón especial utilizado para una mejor navegación dentro de espacios tridimensionales mediante un mango sensible a la presión $(\operatorname{LogiCad} 3 \mathrm{D}, 2000)$. Durante este periodo de tiempo múltiples variaciones de los dispositivos de interacción básicos fueron creados incluyendo una versión específica de dispositivos basados en guantes como el Data Glove de VPL TM (Zimmerman, Lanier, Blanchard, Bryson, \& Harvill, 1987) o el Power Glove de Mattel TM (Quek, 1994). Las diferentes compañías de videojuegos tenían sus propios diseños y orientaciones de gamepad, y comenzó la aparición de dispositivos volante con propósitos experimentales; las simulaciones y videojuegos de arcade comenzaron a ganar audiencia, etc. Durante estos años la principal mejora para una inmersión más profunda fueron las versiones hápticas de los dispositivos ya mencionados recreando el sentido del tacto mediante la aplicación de fuerza, vibraciones o movimientos al usuario.

Estos dispositivos también observaron una gran mejora en la ergonomía. Los equipos de computación en los cuales estos dispositivos eran conectados se volvieron significativamente más pequeños, pero más poderosos y veloces. Los costos de producción se encontraban alrededor de los millones de dólares, lo cual permitió un precio más accesible a los gamepads y ratones permitiendo su producción y venta al público.

\section{Era del Consumidor}

La era del consumidor cubre del 2010 hasta el día de hoy y está fuertemente inspirado en la tecnología de percepción electrónica (Roeber, Bacus \& Tomasi, 2003), tal es el caso del dispositivo Kinect de Microsoft TM (Microsoft, 2013) y la tecnología HeadMounted Display o HMD (Blanchard, Burgess, Harvill, Lanier, Lasko, Oberman, \& Teitel, 1990). La última de estas tecnologías ofrece con la ayuda de sensores detectar la posición y orientación de objetos reales, esta información es utilizable al resto del sistema. El uso más común de esta información es la monitorización de la cabeza y las manos del usuario. Esta tecnología se ofrece desde dos enfoques: óptico o inercial. El primero capta imágenes, a través de cámaras ópticas con reflectores infrarrojos. El segundo obtiene información relativa de la aceleración ejercida por una masa pequeña que inclina al moverse, a través de dispositivos piezoeléctricos sensibles a los cambios de orientación (Duarte, 2018). 
En 2010 uno de los dispositivos de interacción más perceptivos de la historia fue lanzado por Microsoft el Kinect. Este es un dispositivo que posibilita a los usuarios a reconocer e interactuar con una consola sin necesidad de tener contacto físico con un controlador de videojuegos habitual mediante una interfaz natural de usuario que reconoce gestos, comandos de voz, objetos e imágenes (Arce, 2013). La tecnología del Kinect dispone de una cámara con sensores infrarrojos y micrófono multi array capaz de separar voces provenientes de frente al dispositivo de los otros sonidos del ambiente (Microsoft, 2013). Este dispositivo fue creado para videojuegos pertenecientes a la consola casera Xbox 360 pero como resultado de su gran desempeño y mayores posibilidades, se volvió un dispositivo preferido para la interacción en AVs en múltiples campos y diferentes ambientes como por ejemplo rehabilitación física generalizada (Chang, Lange, Zhang, Koenig, Requejo, Somboon \& Rizzo, 2012), navegación de Google Earth (Boulos, Blanchard, Walker, Montero, Tripathy \& Gutierrez-Osuna, 2011) y detección de caídas en hogares de adultos mayores (Stone \& Skubic, 2015).

El año 2016 se vio el lanzamiento de no solo uno sino tres de los más avanzados y ergonómicos dispositivos HMD en la historia. El Oculus Rift TM, HTC Vive TM y PlayStation VRTM. Originalmente la tecnología de HMD se caracterizaba por ser dispositivos muy pesados, para la cabeza del usuario, por lo que había que tenerlos colgados del techo del laboratorio. Las nuevas versiones fueron mejorando, logrando con ello alcanzar dispositivos que permite reproducir imágenes creadas por computadora, muy cercanas a las de los ojos humanos (Arce, 2013). Dos de estos dispositivos fueron ya mejorados con controladores de seguimiento de movimiento, el Oculus Touch de Oculus Rift y los controles de HTC Vive, además el PlayStation VRTM fue complementado con los ya existentes PlayStation Move TM. Estos dispositivos marcaron una revolución para la interacción con los AVs con grandes niveles de inmersión y accesibilidad haciendo al 2016 el año de la RV.

Estos dispositivos fueron creados para el entrenamiento, pero sus aplicaciones en otros campos van más allá de lo que imaginamos. Estos dispositivos están siendo actualmente estudiados e investigados, siendo aplicados lentamente a diferentes campos alrededor del mundo. Las compañías Oculus y HTC se encuentras actualmente enfocando sus esfuerzos en mejorar su tecnología, de tal manera que se puedan crear dispositivos más económicos y precisos que permitan a la realidad virtual ser accesible, para todos como nunca antes lo ha sido y proveerla de una experiencia completamente inmersiva. Dentro del aspecto de la precisión, se espera alcanzar una mejor resolución para cada ojo, y colocar mejores sensores de posición y orientación.

En esta misma era, otro dispositivo llamado The Leap surge en la empresa Leap Motion, la cual deja ver el alcance del futuro de la RV y de la Realidad Aumentada (RA). Esta tecnología se caracteriza por ser un sensor de movimientos, capaz de capturar el movimiento realizado con las manos de los usuarios. El seguimiento manual se ejecuta en procesadores móviles con baja latencia y alta precisión. El artefacto logra una interacción entre el usuario y el ordenador, a través de gestos haciendo posible una nueva experiencia del usuario, la cual difiere con la práctica tradicional donde la experiencia es, a través del teclado y ratón (LeapMotion, 2018).

Un avance complementario con los cascos de RV, que permite acercar más a los usuarios con los EVs son los andadores de RV. Estos se reconocen por ser como caminadoras de 
gimnasio, que permiten a los usuarios desplazarse de forma segura y libre en cualquier dirección, lo que facilita movimientos de $360^{\circ}$. El Virtuix Omni fue la primera opción, con una forma cóncava en donde los pies de los usuarios se apoyan. Los movimientos de los usuarios pueden verse limitados por un arnés de seguridad con el que cuenta, impidiendo que puedan inclinarse. Sin embargo, éste puede evitar cualquier accidente (VirtuixOmni, 2019). Otro andador más ligero fue Cyberith Virtualizer, un nuevo dispositivo con un diseño más ligero. El dispositivo los conforma una alfombra que ser desliza, de acuerdo con los pasos que el usuario realiza sobre la superficie en cualquier dirección (Katvr, 2018).

La mayoría de los dispositivos de este periodo requieren solo de una computadora personal. Su rendimiento experimenta una gran mejora cuando son conectados a un equipo de escritorio equipado con una tarjeta gráfica preparada para RV. Además, su costo de producción fue drásticamente reducido permitiendo así que logren alcanzar al público abierto. Con un precio minorista de 249 dólares para el Kinect, 399 dólares para el Oculus Rift, 499 dólares para el HTC Vive y 299 dólares por el PlayStation VR (PlayStation, 2016), haciendo de la realidad virtual una experiencia alcanzable como nunca antes, y logrando una realidad la inmersión profunda para muchas personas.

En la Figura 1 se presenta una línea de tiempo que cubre las tres eras descritas. En esta Figura 1 se puede observar las invenciones ya mencionadas localizadas en su respectivo período de tiempo, con la excepción de la tecnología de percepción electrónica, debido a que su invención fue previa a su desarrollo llevado a cabo varios años después.

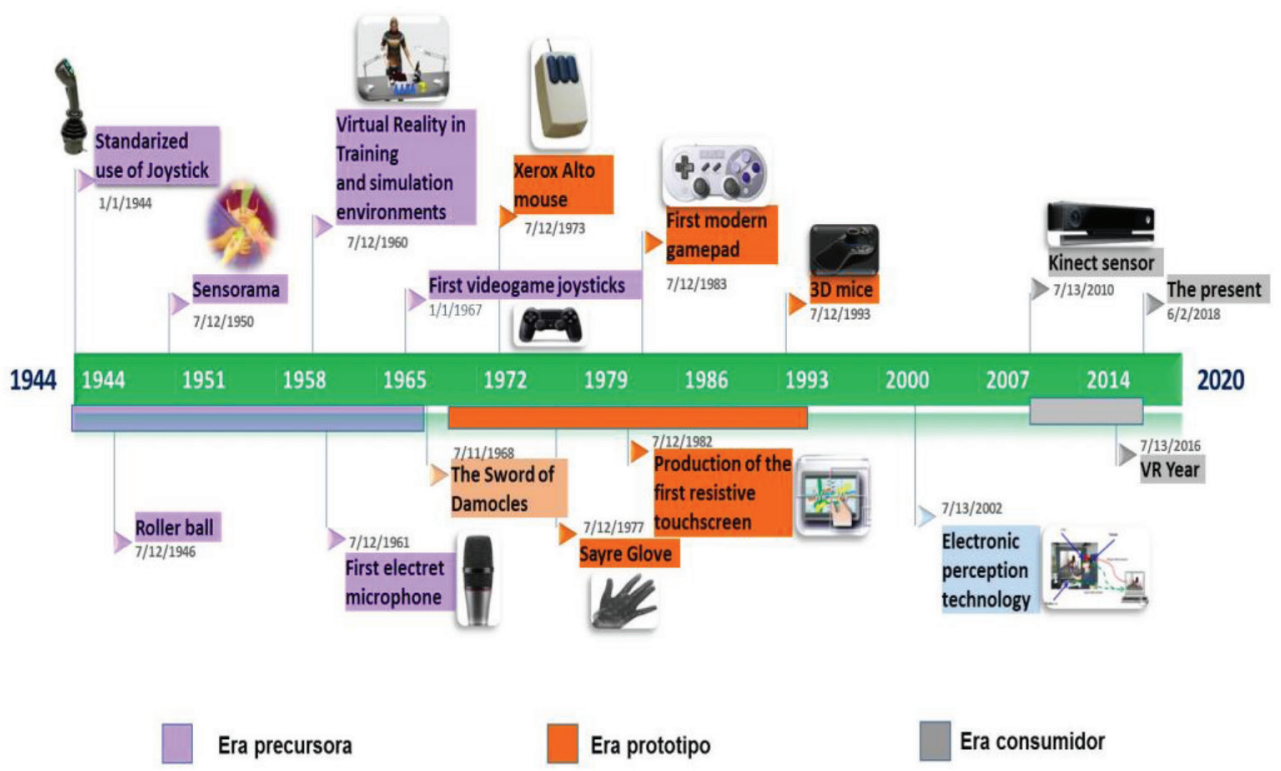

Figura 2 - Eras de los dispositivos de interacción para ambientes virtuales 
La Figura 2 muestra los predecesores de las tecnologías mostradas en la Figura 1, viajando a través de la historia para localizar como cada tecnología evoluciono desde su invención.



Figura 3 - Línea de tiempo de las tecnologías descritas, mostrando su desarrollo a través de los años 


\section{Conclusiones}

Para poder ser parte de la Realidad Virtual con un buen nivel de inmersión, no es suficiente disponer de una herramienta de software, para ello es indispensable contar con un conjunto de dispositivos clave que permitan introducirse en los AVs. Sin duda, el avance de los dispositivos de interacción para los AVs está unido a los avances tecnológicos en el hardware, lo cual ha permitido mayores niveles de inmersión. Esto, expandió la ya abundante posible aplicación de la realidad virtual y otros ambientes virtuales.

Son las aplicaciones de software las encargadas de generar y enviar la información necesaria hacia el equipo de cómputo desde los AVs, pero para llevar a cabo este proceso se requiere un equipamiento adecuado conformado por varios elementos, para montar un sistema completo de Realidad Virtual.

En este trabajo, se identificaron tres épocas utilizando factores de clasificación como los costos de producción y las metas de los dispositivos de interacción.

Para la primera era, puede observarse fácilmente que se requerían una inmensa cantidad de investigación y recursos para desarrollar los diferentes dispositivos de interacción y la mayoría poseían un objetivo de entrenamiento y/o misión militar o aeronáutica.

La determinada segunda era puede caracterizarse por el alto costo requerido para la fabricación de dispositivos de interacción, requiriendo así que múltiples compañías desarrollan sus propios dispositivos, algunos de ellos sirvieron como estándares para múltiples dispositivos en el futuro. Aquí, los objetivos de los AV y sus dispositivos de interacción comenzaron a explorar diferentes campos y fueron exitosamente aplicados a los mismos.

En lo que respecta a la era del Consumidor, puede observarse a los diferentes dispositivos de interacción encontrados en la era anterior, propiamente establecidos y estandarizados, suficiente para establecer un mercado alrededor de ellos, lo cual sirve de evidencia de la reducción drástica en los costos de producción al punto de permitir la existencia de equipos caseros para la realidad virtual con altos niveles de inmersión al alcance de la mano.

En resumen, dentro de este estudio también se analizó, que la evolución tecnológica del hardware para la RV, ha mejorado a través de los años desde diferentes aspectos tales como: rendimiento, costo, ergonomía, resolución, entre otros, llegando a despertar el interés de los fabricantes y de los usuarios. Son muchas las novedades que podríamos esperar a futuro de la Realidad Virtual.

\section{Referencias}

Islas, E., Zabre, E., \& Pérez, M. (2004). Evaluación de herramientas de hardware y software para el desarrollo de aplicaciones de realidad virtual. Bulletin Electrical Research Institute, 28, 61-67.

Arango, J. E., Mazo, J. C., \& Palacio, A. P. (2013). Sistema para rehabilitacion del sindrome del miembro fantasma utilizando interfaz cerebro-computador y realidad aumentada. RISTI-Revista Ibérica de Sistemas e Tecnologias de Informação, (11), 93-106. 
Wadlow, T. A. (1981). The Xerox alto computer. Byte Magazine, 6(9), 58-68.

Meehan, M., Insko, B., Whitton, M., \& Brooks Jr, F. P. (2002). Physiological measures of presence in stressful virtual environments. ACM Transactions on Graphics (TOG), 21(3), 645-652.

Engineering and Technology History Wiki (2015), Ralph Benjamin, an oral history conducted in 2005 by Peter Hill. Hoboken, NJ, USA: IEEE History Center. Retrieved from: https://ethw.org/Oral-History:Ralph_Benjamin.

Naval Research Laboratory (2011). NRL review 2011. Washington, DC: NRL. Retrieved from: https://www.nrl.navy.mil/content_images/2011_NRL_Review.revised.pdf.

Wolf, W. (1997). German Guided Missiles: Henschel HS 293 and Ruhrstahl SD 1400X, (Vol. 53). Merriam Press.

Leung, A. M. (1990). U.S. Patent No. 4,924,216. Washington, DC: U.S. Patent and Trademark Office.

Huhtamo, E. (2007). Slots of fun, slots of trouble. Artnodes, 7.

Furness III, T. A. (1986). The super cockpit and its human factors challenges. In: Proceedings of the Human Factors Society Annual Meeting (Vol. 30, No. 1, pp. 4852). Los Angeles, CA: SAGE Publications.

Encicplopedia Británica. (2019). Retrieved from: https://www.britannica.com/ technology/virtual-reality.

Arce, C. (2013). Realidad Aumentada. Retrieved from: http://jeuazarru. com/ wpcontent/uploads/2014/10/RA2013. pdf.

Heilig, M. L. (1962). U.S. Patent No. 3,050,870. Washington, DC: U.S. Patent and Trademark Office.

Heilig, M. (1998). Beginnings: sensorama and the telesphere mask. In: Digital illusion (pp. 343-351). ACM Press/Addison-Wesley Publishing Co.

Martín-Herrera, B. (2012). El móvil en la educación: un nuevo paradigma. Ventajas y desventajas de su uso.

Sutherland, I. E. (1965). The ultimate display. Multimedia: From Wagner to virtual reality, (pp. 506-508).

Roeber, H., Bacus, J., \& Tomasi, C. (2003). Typing in thin air: the canesta projection keyboard-a new method of interaction with electronic devices. In: CHI'O3 extended abstracts on Human factors in computing systems (pp. 712-713). ACM.

Chang, C. Y., Lange, B., Zhang, M., Koenig, S., Requejo, P., Somboon, N., ... \& Rizzo, A. A. (2012). Towards pervasive physical rehabilitation using Microsoft Kinect. In: Pervasive Computing Technologies for Healthcare (PervasiveHealth), 2012 6th International Conference on (pp. 159-162). IEEE.

Boulos, M. N. K., Blanchard, B. J., Walker, C., Montero, J., Tripathy, A., \& GutierrezOsuna, R. (2011). Web GIS in practice X: a Microsoft Kinect natural user interface for Google Earth navigation. 
Stone, E. E., \& Skubic, M. (2015). Fall detection in homes of older adults using the Microsoft Kinect. IEEE journal of biomedical and health informatics, 19(1), 290-301.

Boas, Y. A. G. V. (2013). Overview of virtual reality technologies. In: Interactive Multimedia Conference (Vol. 2013).

Sutherland, I. E. (1968). A head-mounted three-dimensional display. In: Proceedings of the December 9-11, 1968, fall joint computer conference, part I (pp. 757-764). ACM.

Sturman, D. J., \& Zeltzer, D. (1994). A survey of glove-based input. IEEE Computer graphics and Applications, 14(1), 30-39.

Duarte, M., C. (2018). Hacia el espacio. Retrieved from: https://haciaelespacio.aem. gob.mx/revistadigital/articul.php?interior $=796$.

VirtuixOmni. (2019). Retrieved from: http://www.virtuix.com/.

Katvr. (2018). Retrieved from: http://katvr.com/about/.

LeapMotion. (2019). Retrieved from: https://developer.leapmotion.com/\#101.

Blanchard, C., Burgess, S., Harvill, Y., Lanier, J., Lasko, A., Oberman, M., \& Teitel, M. (1990, February). Reality built for two: a virtual reality tool. ACM SIGGRAPH Computer Graphics 24(2), 35-36.

Wolf, M. J. (1997). Inventing space: Toward a taxonomy of on-and off-screen space in video games. Film Quarterly (ARCHIVE), 51(1), 11.

Zimmerman, T. G., Lanier, J., Blanchard, C., Bryson, S., \& Harvill, Y. (1987). A hand gesture interface device. ACM SIGCHI Bulletin 18(4), 189-192.

Quek, F. K. (1994). Toward a vision-based hand gesture interface. In: Virtual reality software and technology (pp. 17-31).

LogiCad3D. (2000). Magellan/SpaceMouse Classic/Plus/Plus XT.: User's Manual. Seefeld, Germany: LogiCad3D.

3DConnexion. (2003). SpaceTraveler ${ }^{\mathrm{TM}}$ Small size. Big performance: User's Manual. Seefeld, Germany: 3DConnexion. Retrieved from: http://www.surface3d.com.tw/ spacetraveler/SpaceTraveler_En.pdf.

3DConnexion. (2007). SpaceExplorer ${ }^{\mathrm{TM}}$ Navigate Your 3D World ${ }^{\mathrm{TM}}$ : User's Manual. Seefeld, Germany: 3DConnexion. Retrieved from: http://www.crystaltech.ca/Pdfs/ SpaceNavigator_Datasheet.pdf.

3DConnexion. (2018). Retreived from: https://www.3dconnexion.com/service/drivers. html.

Microsoft. (2013). Xbox one and kinect sensor product manual: Product's Manual. Redmond, WA, USA: Microsoft.

Sony. PlayStation. (2016). PlayStation ${ }^{\circledR}$ Camera: Instruction Manual. Zeventem, Belgium: Sony. Retrieved from: https://www.playstation.com/es-mx. 